\title{
Choriocarcinoma (stage IV) despite two normal dilation and curettages: $A$ case report
}

\begin{abstract}
Choriocarcinoma is a highly metastatic subtype of gestational trophoblastic neoplasia (GTN) following pregnancy. It can arise from any type of pregnancy $50 \%$ occur after term pregnancies, $25 \%$ after molar pregnancies, and $25 \%$ after early pregnancy loss and ectopic pregnancies) (Soper, 2006).

This case report describes an interesting diagnostic conundrum with normal histology findings on two separate endometrial dilation and curettages (D\&C) and a unilateral oophorectomy in the context of high clinical suspicion for molar pregnancy that progressed to stage IV choriocarcinoma with brain and lung metastasis.

The patient presented to our hospital nearly aphasic but would only say "molar pregnancy". Due to her brain metastasis and worsening intraparenchymal hematoma, she underwent craniotomy confirming the diagnosis. After stabilization, she was transferred to a quaternary care center to complete chemotherapy with etoposide, methotrexate, actinomycin, cyclophosphamide, and vincristine and had a good response.

At this time, there is no clear explanation for normal histology on two separate D\&Cs and a unilateral oophorectomy in the context of a classic case of choriocarcinoma with progression from a molar pregnancy. It reminds us as providers that medicine remains an imperfect science and requires astute clinical judgement to properly treat patients when diagnostic data such as pathology findings and lab values appear to be incongruent with the larger clinical picture.
\end{abstract}

Keywords: choriocarcinoma, normal dilation and curettage, gestational trophoblastic neoplasia
Volume 12 Issue 5 - 202I

\author{
Bijan Morshedi, Laura Haworth, Traci Ito \\ Department of Obstetrics and Gynecology, EVMS, USA
}

Correspondence: Bijan Morshedi, MD, Eastern Virginia Medical School (EVMS) Department of Obstetrics and Gynecology, USA, Tel (757) 446-7979, Email bm8wr@virginia.edu

Received: October 05, 202। | Published: October 20, 202 |

\section{Introduction}

Choriocarcinoma is a highly metastatic subtype of gestational trophoblastic neoplasia (GTN) following pregnancy. It can arise from any type of pregnancy (50\% occur after term pregnancies, $25 \%$ after molar pregnancies, and $25 \%$ after early pregnancy loss and ectopic pregnancies). ${ }^{1}$ Diagnosis of choriocarcinoma is unique in that it is one of the few cancers that does not require histology findings to meet diagnostic criteria. However, when histology is available from endometrial samples or metastasis, it is typically characterized by the absence of chorionic villi, presence of hyperplastic or anaplastic syncytiotrophoblastic and cytotrophoblasts, diffuse hemorrhage, tissue necrosis, lymphovascular invasion, marked cytologic pleomorphism, nuclear enlargement, and brisk mitotic activity. ${ }^{2-4}$

This case report describes an interesting diagnostic conundrum with normal histology findings on two separate endometrial dilation and curettages (D\&C) in the context of high clinical suspicion for molar pregnancy that progressed to stage IV choriocarcinoma with brain and lung metastasis.

\section{Case report}

The patient is an 18-year-old G3P2002 with a history of two prior spontaneous vaginal deliveries complicated by limited prenatal care. She presented in April to an outside ED seeking confirmation of pregnancy, with last menstrual period four weeks prior and a positive home urine pregnancy test (UPT). Qualitative UPT was positive and the patient was referred to follow up with a local OBGYN. The patient reported transportation difficulties, however, and was lost to follow up.
Three months later the patient represented to an ED with vaginal bleeding and a beta-human chorionic gonadotropin (b-HCG) of 1 million. Transvaginal ultrasound (TVUS) showed an enlarged uterus $(21 \times 17 \times 13 \mathrm{~cm})$ with heterogenous intrauterine solid and cystic components suggestive of molar pregnancy versus incomplete early pregnancy loss. A chest x-ray was completed given concern for malignant potential from her suspected molar pregnancy, and was negative for lesions. A D\&C produced an $85 \mathrm{~g}$ tissue sample. No fetal parts were grossly identified and the sample otherwise included multiple tan-pink, red, brown, hemorrhagic, spongy, friable soft tissue fragments and blood clots. A second $27 \mathrm{~g}$ specimen consisting of sharp currettings included only tan-pink spongy friable hemorrhagic soft tissue fragments with blood clots. Both histologic specimens included decidualized tissue, without chorionic villi or fetal tissue, making them incongruent with the high clinical suspicion for molar pregnancy. Given this diagnostic conundrum, additional sections were examined. Nevertheless, the same unremarkable findings were noted. Post-D\&C TVUS demonstrated that the contents were effectively removed, with a markedly reduced endometrial thickness of $10 \mathrm{~mm}$, and an endometrial cavity measuring $7 \times 5 \times 4 \mathrm{~mm}$ with a small amount of fluid and blood. An injection of depo-provera was provided and she was discharged to home with recommendation for close clinic follow up. Unfortunately, the patient was again lost to follow up.

Five months after initial presentation, the patient presented to the ED for the third time with continued vaginal bleeding and a b-HCG of 58,000 . Clinical suspicion for molar pregnancy remained very high per review of records despite incongruent histology findings. TVUS was repeated and the patient underwent a repeat D\&C due to presumably continued uterine enlargement with intracavitary findings 
on the TVUS concerning for molar pregnancy. Tissue samples from this second D\&C were negative for definitive trophoblastic elements and chorionic villi. Endometrial samples revealed inflamed and decidualized endometrium intermixed with blood clot. Sharp endometrial curettage revealed detached fragments of decidualized endometrium, benign endocervical glandular cells, and cervical squamous epithelium intermixed with abundant blood clot. These findings were again incongruent with the highly suspected molar pregnancy and also choriocarcinoma. The patient recovered well and was discharged with scheduled outpatient appointment, however, was lost to follow up.

One month later the patient presented to the ED for the fourth time with abdominal pain. TVUS demonstrated a slightly enlarged uterus measuring $11 \times 8 \times 7 \mathrm{~cm}$, a normal endometrial thickness of $8.4 \mathrm{~mm}$, and no intrauterine pregnancy visualized. She reported experiencing regular periods at this time, however, her b-HCG was 124,000. The patient was recommended to follow up in the clinic and was discharged home and lost to follow up.

The patient then presented nine months later to a local clinic after reporting missing her period the prior month. Her b-HCG was ">10,000" and prenatal labs were unremarkable. One month later a follow up transabdominal ultrasound showed a diffusely abnormal uterus with multiple cystic changes and a complex hypervascular mass like lesion without a fetal pole or yolk sac. Further inpatient evaluation was recommended but the patient declined and was lost to follow up.

Later that month, the patient presented to our ED for the first time after being found to be minimally conversive at home. Per her family member, the patient had been largely asymptomatic with minor nausea prior to this acute change in mental status. The patient answered yes/no for all questioning with the exception of repeating the phrase "molar pregnancy." Complete blood count, basic metabolic panel, and Covid-19 tests were normal. A qualitative UPT was negative, thyroid stimulating hormone was $0.01 \mathrm{mIU} / \mathrm{L}$ with free $\mathrm{T} 4$ of $5.4 \mathrm{ng} / \mathrm{dL}$, and urine drug screen was unremarkable. Vital signs were stable aside from sinus tachycardia in the low 100s. Within two hours the patient experienced a generalized tonic clonic seizure. CT head without contrast revealed $3.6 \times 3.3 \times 3.8 \mathrm{~cm}$ left frontal intraparenchymal hematoma with significant vasogenic edema and a left to right midline shift (Figure 1). TVUS showed a complex intrauterine hypervascular structure. Abdominal CT reveled a markedly enlarged and heterogeneous appearing uterus concerning for malignant process of uterine etiology (Figures 2). An additional large multiloculated cystic structure was identified, centered at the left adnexa with differential diagnosis of complex ovarian cystic mass vs ovarian tumor vs hydrosalpinx. CT chest revealed innumerable nodules throughout the bilateral lungs concerning for metastatic disease (Figure 3). A quantitative b-HCG was $>2.5$ million with normal cancer markers (CEA of $1.9 \mathrm{ng} / \mathrm{mL}, \mathrm{CA}-12526.80 \mathrm{U} / \mathrm{mL}$, AFP $3.7 \mathrm{ng} / \mathrm{mL}$ ), confirming the diagnosis of choriocarcinoma.

The patient was transferred to the Neuro ICU for stabilization. In the Neuro ICU, she indicated severe headaches and had progressive neurological deficits over several days. Serial CT scans demonstrated a worsening intraparenchymal left frontal lobe hematoma. Neurosurgery elected to proceed with a left craniotomy with excision of a suspected cranial arteriovenous malformation.

The mass removed was histologically confirmed as choriocarcinoma. Removal of the mass luckily was not accompanied by marked bleeding. The next day the patient regained significant neurologic function and was able to speak in full sentences. A pelvic exam was performed with patient consent showing bilateral pelvic and vaginal metastasis. On hospital day seven she was transferred to a quaternary care center to receive chemotherapy with etoposide, methotrexate, actinomycin, cyclophosphamide, and vincristine. On chart review, the patient responded well with appropriate decline in b-HCG. It is likely she will make a full recovery and maintain her fertility. Her most recent b-HCG was $42 \mathrm{mIU} / \mathrm{mL}$.

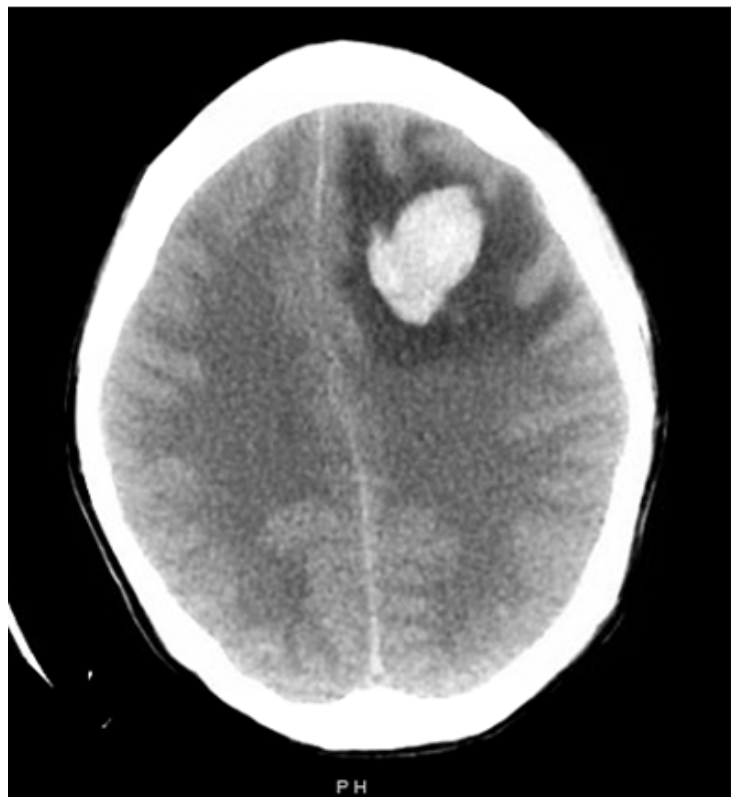

Figure I CT head without contrast revealing a $3.6 \times 3.3 \times 3.8 \mathrm{~cm}$ left frontal intraparenchymal hematoma with significant vasogenic edema and a left to right midline shift and subsequently confirmed brain metastasis from choriocarcinoma.

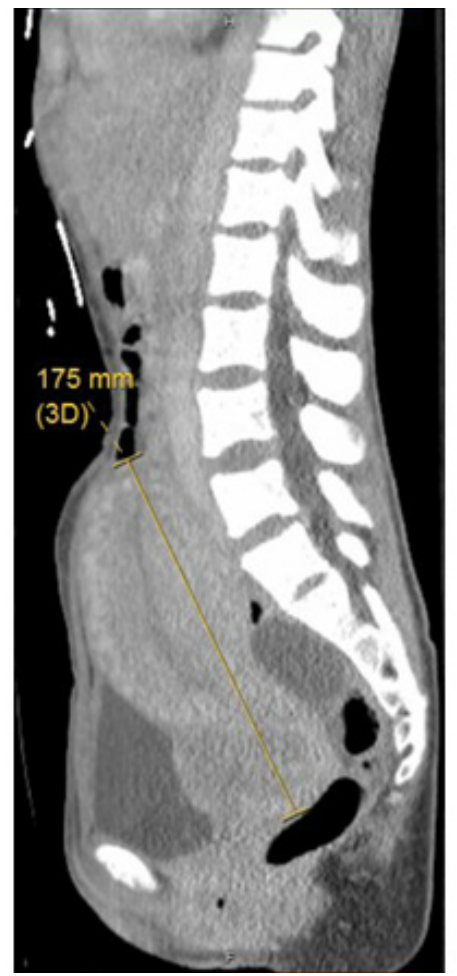

Figure 2 Midsagittal view of the uterus. 


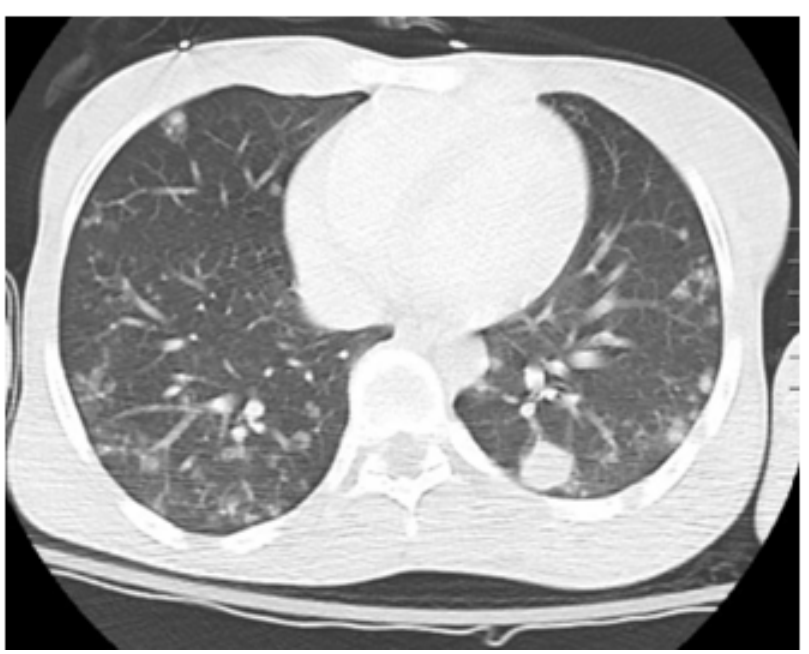

Figure $3 \mathrm{CT}$ chest with contrast concerning for multiple lung metastasis from choriocarcinoma.

Given the unexpectedly normal histologic findings of this patent's case, the permanent tissue samples from the prior D\&Cs in the preceding year were reexamined by the Division of Gynecologic Pathology at the original institution through their Intradepartmental Quality Assurance Conference. Their final interpretation was as follows: H\&E sections demonstrated fragments of decidua with acute inflammatory changes, fibrin, and hemorrhage. Chorionic villi were not identified, which precludes the evaluation for a hydatidiform mole. Within the fragments of decidua is implantation-type intermediate trophoblast (highlighted by an hPL stain), which involve the decidua in their usual pattern as opposed to forming confluent sheets of cells, confirming the presence of an intrauterine gestation. A p63 stain highlights rare chorionic-type intermediate trophoblast. An hCG immunostain is non-contributory, cytokeratin AE1/AE3 highlights epithelial elements and trophoblast, and a Ki-67 stain is not increased (with foci up with up to a $10 \%$ proliferative index). While there are features an exaggerated implantation site, definite evidence of a malignant trophoblastic proliferation cannot be identified, including but not limited to choriocarcinoma. All of the Pathologists in attendance agreed with the above diagnosis.

\section{Discussion}

This case report highlights the only reported case of FIGO stage IV choriocarcinoma that likely progressed from persistent GTD, despite two separate normal D\&Cs incongruent with both molar pregnancy and choriocarcinoma at time of disease progression. Possibilities that could explain normal D\&C pathology findings include inadequate surgical sampling, inadequate pathology sampling, and primary choriocarcinoma (a rare but reported phenomenon). Given that 100 grams of tissue was extracted from the first D\&C with reduction of the uterus from 20 weeks to 7 weeks size, inadequate surgical sampling is unlikely. Also given that 6 cassettes were initially examined by the pathologist, with a request for additional tissue specimens and a review of additional specimens from the initial tissue sample by two different attending pathologists, an inadequate pathology sampling is unlikely. Lastly, it is possible the patient had a primary choriocarcinoma. While not detailed above, in the course of her workup the patient underwent a right oophorectomy for an enlarged adnexal mass, which was found to be benign hemorrhagic corpus luteal cyst. The likelihood of an unrelated, primary choriocarcinoma coincidentally following an incomplete early pregnancy loss and unilateral oophorectomy is extremely low. This would leave the most common sites of origin for this choriocarcinoma to be the myometrium or serosa of the uterus (preventing sampling during a D\&C), the lymphatic or vascular system in the pelvis, or involvement of the remaining fallopian tube or ovary.

Another interesting point in the case is the negative UPT at the patient's final ED presentation where choriocarcinoma was diagnosed. While the quantitative b-HCG was $>2$ million, the initial qualitative UPT was negative. Had the mostly aphasic patient been unable to voice "molar pregnancy" the decision for a quantitative b-HCG might have been significantly delayed, furthering muddying the diagnostic picture. This false negative result has been documented as the hook effect, which can affect enzyme-linked immunosorbent assays when ligands are present in high enough concentrations to saturate both the fixed antibodies and the enzyme linked detection antibodies. This prevents the two antibodies from complexing with each other, resulting in a false negative test. This typically occurs for UPT at b-HCG concentrations of $500,000 \mathrm{mIU} / \mathrm{mL}$ or greater, which can occur in GTD, as it did with this patient. To prevent the hook effect, serum obtained from the patient should be diluted in clinically appropriate cases if the first result is negative, or a quantitative b-HCG measurement can be performed. ${ }^{5}$

At this time, there is no clear explanation for two negative D\&Cs in the context of a classic case of choriocarcinoma with progression from a molar pregnancy. It reminds us as providers that medicine remains an imperfect science and requires astute clinical judgement to properly treat patients when diagnostic data such as pathology findings and lab values appear to be incongruent with the larger clinical picture. Physicians at several institutions in this patient's case were correct in continuing to have a high suspicion for molar pregnancy and concern for malignant potential despite incongruent histology findings. It also further serves to remind us that the diagnosis of choriocarcinoma while supported by histology findings, is not required.

\section{Acknowledgments}

None.

\section{Funding}

None.

\section{Conflicts of interest}

The authors did not report any potential conflicts of interest.

\section{References}

1. Soper JT. Gestational trophoblastic disease. Obstet Gynecol. 2006;108(1):176-187.

2. Heller DS. Update on the pathology of gestational trophoblastic disease. APMIS. 2018;126(7):647-654.

3. Hou MM, Xu L, Qie MR. Postmolar choriocarcinoma after an interval of 7 years: Case report and literature review. Gynecol Minim Invasive Ther. 2017;6(4):207-210.

4. Hui P. Gestational trophoblastic tumors: A timely review of diagnostic pathology. Arch Pathol Lab Med. 2019;143(1):65-74.

5. Winder AD, Mora AS, Berry E, et al. The "hook effect" causing a negative pregnancy test in a patient with an advanced molar pregnancy. Gynecol Oncol Reports. 2017;21:34-36. 